\title{
CRECIMIENTO INICIAL DE 23 PROCEDENCIAS Y 196 FAMILIAS DE Eucalyptus camaldulensis Denh EN CUATRO SITIOS DE LA ZONA CENTRAL DE CHILE
}

Braulio Gutiérrez C. (*)

Patricio Chung G. (*)

\section{RESUMEN}

So analiza la variación del crecimiento inicial y supervivencia de 23 procedencias y 196 familias de Eucalyptus camaldulensis Denh establecidas on cuatro ensayos de procedencia y progenie en la zona central de Chile (32 - 35' Lat. Sur)

Se concluye que existen diferencias significativas de crecimiento a nivel de procedencias y progenies, destacándose aquellas de los lagos Hindmarsh y Albacutya como las más interesantes.

Palabras claves: Eucalyptus camaldulensis, procedencias, progenies.

\section{ABSTRACT}

Four Eucalyptus camaldulensis Denh provenance and progenie trials established in the central zone of Chile ( $32-35^{\circ}$ L.S.) were assesed. In those trials, the survival and early growth of 196 families and 23 provenances were analyzed.

Significant growth differences among provenances and families were obtained. Best growth results are achieved by the Lake Hindmarsh and Lake Albacutya provenances.

Key words: Eucalyptus camaldulensis, provenances, families. 


\section{INTRODUCCION}

La importancia del género Eucalyptus se ha incrementado a nivel mundial, llegando a constituirse en uno de los más productivos cultivos forestales. Esta situación obedece a la gran diversidad que presenta este género en cuanto a número de especies y procedencias, asi como también a la amplia variabilidad genética que exhibe a nivel de especies, procedencias, progenies, e incluso individuos dentro de las progenies, lo que en la práctica ha posibilitado su establecimiento con fines productivos sobre una muy amplia gama de sitios.

En Chile, el Instituto Forestal ha realizado desde 1962 múltiples ensayos de introducción de especies, que han permitido individualizar a aquellas que brindan las mejores posibilidades de uso en las distintas zonas del pais y de cuyos resultados se desprende que $\mathbf{E}$. camaldulensis es una de las especies más apropiadas para forestar las zonas semiáridas de éste (Infor-Corfo, 1986)

Las regiones semiáridas se caracterizan por presentar un frágil equilibrio ecológico y por estar sometidas a una constante presión antrópica derivada de la demanda por combustibles leñosos, los cuales en forma de carbón y leña son extraidos desde las escasas formaciones vegetales existentes. Este fenómeno, manifestado históricamente, ha contribuido a disminuir cada vez más la cubierta vegetal y a aumentar la degradación de los suelos. Por lo mismo, el establecimiento de una cubierta vegetal es una necesidad imperiosa, tanto para fines de producción como de protección, perfilándose el E. camaldulensis como una especie muy favorable para satisfacer ambos requisitos.

E. camaldulensis presenta una extensa área de distribución natural, encontrándose presente en todos los estados australianos con excepción de Tasmania (Tumbull, 1973). Debido a este amplio rango de distribución, la especie presenta una gran variabilidad genética, a la cual se suma la variabilidad que existe entre individuos que crecen en sitios de condiciones similares. Por lo mismo, la selección de las mejores procedencias, progenies o individuos es de particular importancia, ya que existen marcadas variaciones en el hábito de crecimiento de los árboles (tasa de crecimiento, rectitud de fuste) y en su plasticidad ecológica para adaptarse a distintas condiciones de 
sitio o resistir a los efectos de heladas, sequias, plagas, etc. (Infor, 1992).

Por otra parte, E. camaldulensis es una de las especies más difundidas de su género, estimándose que existen más de $\mathbf{5 0 0}$ mil hectáreas de plantaciones fuera de Australia, principalmente en España y Marruecos (FAO, 1981). En estos y otros países se han realizado estudios que demuestran la existencia de un grado considerable de variación intraespecífica y concluyen que la selección de las procedencias de semillas más adecuadas para la forestación de un lugar determinado, puede conducir a importantes ganancias de crecimiento en las plantaciones (Bellefontaine et. al, 1979; Eldrige y Cromer, 1987; Lacaze, 1977; cit por Barros, 1990).

Efectivamente, en Nigeria se ha llegado a triplicar y en Israel a octuplicar el rendimiento volumétrico de las plantaciones mediante la adecuada selección de procedencias. Por su parte en Marruecos, el Servicio Forestal ha reemplazado las procedencias locales de E. camaldulensis por una de Lake Albacutya, Victoria, Australia, con lo cual espera incrementar los rendimientos volumétricos de las plantaciones en más del $100 \%$ (Bellefontaine et. al, 1979; Knockaert, 1984; cit por Barros, 1990).

En Chile, gran parte de la variabilidad genética del E. camaldulensis se encuentra representada en los ensayos de procedencias y progenies que el Instituto Forestal ha establecido desde 1989, en el marco del proyecto Mejoramiento Genético de Eucalyptus y en ensayos de procedencias establecidos anteriormente como parte de los trabajos de introducción de especies, con el objeto de conocer y explotar la variabilidad de la especie y mejorar la calidad y productividad de sus plantaciones.

En este sentido, el presente trabajo representa una evaluación preliminar de la variabilidad en crecimiento inicial de $E$. camaldulensis, como una forma de estimación de las posibilidades de mejoramiento que la base genética existente puede generar. 


\section{OBJETIVO}

Evaluar la variabilidad en crecimiento inicial de E. camaldulensis e individualizar las procedencias y progenies que se perfilan como las más adecuadas para maximizar la producción volumétrica en las plantaciones que se establezcan en la zona central de Chile.

\section{MATERIAL Y METODO}

Para dar cumplimiento al objetivo enunciado se analizaron los valores de supervivencia, diámetro de cuello (d), altura total $(\mathrm{H})$ y del indice $d^{2} \mathrm{H}$, el cual combina a los anteriores y es un buen estimador del crecimiento en volumen (Infante y Prado, 1989; 1991).

Las variables provienen de la tercera evaluacion, correspondiente al segundo año después del establecimiento, de cuatro ensayos de procedencia-progenie de E. camaldulensis.

\section{Ensayos considerados}

Los ensayos contemplados en este estudio son los de Longotoma, Mel-Mel, Tantehue y La Paila (Cuadro $N^{\circ} 1$ ), los cuales se encuentran comprendidos entre los 32 y $35^{\circ}$ de latitud Sur.

Los cuatro ensayos fueron establecidos sobre sitios planos a ligeramente ondulados, con una intensiva preparación de suelo, y bajo un diseño experimental conocido como bloques de familias compactas o parcelas divididas. En esta estructura la parcela principal está constituida por la procedencia, dentro de la cual se distribuyen al azar las progenies en subparcelas de cuatro plantas cada una. Cada procedencia (parcela principal) cuenta con diez repeticiones, constituyendo igual número de bloques, los que en su conjunto se encuentran rodeados por dos hileras de aislación. 


\section{CUADRO N 1}

UBICACION Y CARACTERIZACION DE LOS ENSAYOS CONSIDERADOS

\begin{tabular}{|l|c|c|c|c|}
\hline Ensayo & Mel-Mel & Longotoma & Tantehue & La Paila \\
\hline Región & $\mathrm{V}$ & $\mathrm{V}$ & $\mathrm{RM}$ & $\mathrm{V}$ \\
Provincia & Valparaiso & Petorca & Santiago & Colchagua \\
Comuna & Casablanca & La Ligua & Melipilla & Lolol \\
Latitud & $33^{\circ} 23^{\prime} \mathrm{S}$ & $32^{\circ} 21^{\prime} \mathrm{S}$ & $33^{\circ} 32^{\prime} \mathrm{S}$ & $34^{\circ} 41^{\prime} \mathrm{S}$ \\
Longitud & $71^{\circ} 24^{\prime} \mathrm{W}$ & $71^{\circ} 26^{\prime} \mathrm{W}$ & $71^{\circ} 14 \mathrm{~W}$ & $71^{\circ} 30^{\circ} \mathrm{W}$ \\
Altitud & $250 \mathrm{msnm}$ & $58 \mathrm{msnm}$ & $240 \mathrm{msnm}$ & $300 \mathrm{msnm}$ \\
Ano de establecimiento & 1989 & 1989 & 1991 & 1991 \\
Precipitación media anual & $455 \mathrm{~mm}$ & $308 \mathrm{~mm}$ & $419 \mathrm{~mm}$ & $398 \mathrm{~mm}$ \\
Temperatura media anual & $13,3^{\circ} \mathrm{C}$ & $14,3^{\circ} \mathrm{C}$ & $13,6^{\circ} \mathrm{C}$ & - \\
Periodo seco (Nं de meses) & $7-9$ & $7-9$ & 8 & - \\
\hline
\end{tabular}

\section{Antecedentes de la colección de semillas}

La colección de semillas contemplada en los ensayos es una muestra que representa gran parte del area de distribución natural de la especie (Figura $\mathrm{N}^{\circ}$ 1), encontrándose caracterizada en el Cuadro $N^{\circ} 2$. Esta colección se encuentra individualizada según lugar de origen (procedencia) y árbol madre (progenie), completando un total de 23 procedencias y 196 progenies, a las que se agregan como testigos dos procedencias nacionales: La Ligua y Lolol.

La cantidad de plantas obtenidas a partir de las muestras de semillas disponibles no permitió incluir a todas las progenies en cada uno de los ensayos, presentándose en el Cuadro $\mathrm{N}^{0} 3$ las procedencias y el número de progenies contempladas en cada uno de ellos. 


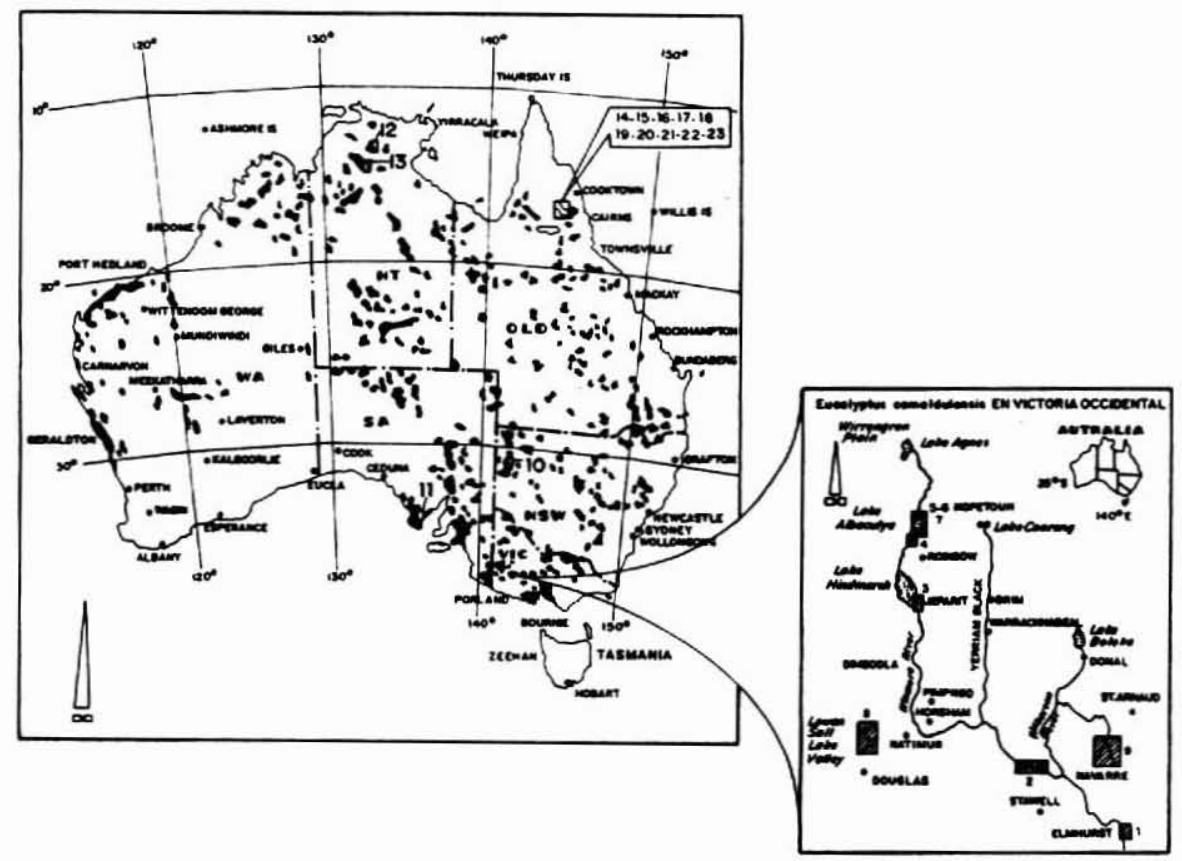

Figura $N^{\circ}$ 1. DISTRIBUCION NATURAL DE E.CAMALDULENSIS Y UBICACION DE LAS PROCEDENCIAS CONSIDERADAS

Fuente: Elaborado en division Silvicultura, INFOR, con antecedentes de Thomson y Merwin, 1985 y Doran, 1985. 


\section{Cuadro $N^{\circ} 2$}

CARACTERIZACION DE LAS PROCEDENCIAS DE E. Camaldulensis CONSIDERADAS EN EL ENSAYO

\begin{tabular}{|c|c|c|c|c|c|c|c|c|c|c|}
\hline \multirow[t]{2}{*}{$\begin{array}{l}\text { Proces } \\
\text { dencia }\end{array}$} & \multirow[t]{2}{*}{$\begin{array}{l}\text { Codigo } \\
\text { CSIRO }\end{array}$} & \multirow[t]{2}{*}{ N Prog. } & \multirow{2}{*}{\multicolumn{2}{|c|}{ Lugar de origen }} & \multicolumn{2}{|c|}{ Temperatun } & \multirow{2}{*}{$\begin{array}{c}\text { Precipiteción } \\
\mathbf{3}(\mathrm{mm})\end{array}$} & \multirow{2}{*}{$\begin{array}{l}\text { Altitud } \\
\text { (msnm) }\end{array}$} & \multirow{2}{*}{$\begin{array}{c}\text { Latitud } \\
\mathbf{S}\end{array}$} & \multirow{2}{*}{$\begin{array}{c}\text { Longitud } \\
\mathbf{W}\end{array}$} \\
\hline & & & & & $1(0)$ & $2(0)$ & & & & \\
\hline 1 & 15021 & 8 & Wimmen R-Elmhurst & VIC & 8 & 19 & 617 & 325 & $37 \cdot 13^{\prime}$ & $143^{-16^{\prime}}$ \\
\hline 2 & 15022 & 12 & Wimmen R-Gienorchy & VIC & 9 & 22 & 448 & 170 & $36.55^{\prime}$ & $142.40^{\circ}$ \\
\hline 3 & 15024 & 10 & Labe Hindmersh-SE & VIC & 8 & 22 & 419 & 75 & $36.08^{\prime}$ & $141.58^{\prime}$ \\
\hline 4 & 15028 & 13 & Outlot Crook-Rainbow & VIC & 8 & 22 & 419 & 75 & $35 \cdot 49^{\prime}$ & $141.56^{\prime}$ \\
\hline 5 & 15027 & 12 & Lale Albecutya-S & VIC & 8 & 22 & 419 & 70 & $35.48 '$ & $141.58^{\prime}$ \\
\hline 6 & 15028 & 11 & Lales Albacutya-E & VIC & 8 & 22 & 419 & 70 & $35.46^{\prime}$ & $142.00^{\circ}$ \\
\hline 7 & 15029 & 12 & Lake Albacutya-N & VIC & 8 & 22 & 419 & 70 & $35.42^{\prime}$ & $141.57^{\prime}$ \\
\hline 8 & 15037 & 10 & Lowan Valloy-Saline & VIC & 9 & 22 & 448 & 135 & $35.50^{\prime}$ & $141.50^{\prime}$ \\
\hline 9 & 15039 & 10 & Avon Riner-Havarre & VIC & 8 & 19 & 617 & 280 & $36.52^{\prime}$ & $143.11^{\prime}$ \\
\hline 10 & $151 \%$ & 10 & Umbenumberle Creek & NSW & 12 & 27 & 254 & 210 & $31.53^{\circ}$ & $141.13^{\prime}$ \\
\hline 11 & 10885 & 10 & $24 \mathrm{~km}$ from Port Lincoln & SA & 12 & 21 & 486 & 90 & $3435^{\prime}$ & $135.38^{\prime}$ \\
\hline 12 & 13923 & 12 & Katherine & NT & 20 & 34 & 952 & 95 & $1429^{\prime}$ & $132.15^{\prime}$ \\
\hline 13 & 14517 & 8 & Katherine & NT & 20 & 34 & 952 & 95 & $1426^{\prime}$ & $132 \cdot 18^{\prime}$ \\
\hline 14 & 14238 & 10 & Eccles CK NE Pottord & $a D$ & 14 & 26 & 800 & 500 & $17 \cdot 17^{\prime}$ & $145.03^{\prime}$ \\
\hline 15 & $\begin{array}{l}14264 \\
14268\end{array}$ & 5 & Pinnacle Ck NE Pettord & $a D$ & 14 & 26 & 800 & 460 & $17 \cdot 13^{\prime}$ & $145.01^{\prime}$ \\
\hline & 14272 & & & & & & & & & \\
\hline 16 & 14291 & 5 & Mishap Ck NE Potford & aco & 14 & 26 & 800 & 520 & $17 \cdot 11$ & $145.07^{\prime}$ \\
\hline & 14296 & & & & & & & & & \\
\hline 17 & 14297 & 5 & Eureka Ck NE Potiord & QLD & 14 & 26 & 800 & 520 & $17 \cdot 15^{\prime}$ & $145.05^{\prime}$ \\
\hline 18 & 14311 & 10 & Wales Siding E Pottord & QLD & 14 & 26 & 873 & 780 & $17 \cdot 22$ & $145.12^{\prime}$ \\
\hline & 14337 & & & & & & & & & \\
\hline 19 & 14344 & 5 & Emu Ck E Pottord & QLD & 14 & 26 & 800 & 460 & $17 \cdot 21^{\prime}$ & $14457^{\prime}$ \\
\hline & 14348 & & & & & & & & & \\
\hline 20 & 14357 & 5 & Emu/gibbs CK SE Pettord & QLD & 14 & 26 & 1.116 & 500 & $17 \cdot 25$ & $145.02^{\prime}$ \\
\hline & 14361 & & & & & & & & & \\
\hline 21 & 14362 & 5 & Gibbs Ck ESE Petiord & QLD & 14 & 26 & 873 & 700 & $17 \cdot 25$ & $145 \cdot 11^{\prime}$ \\
\hline & 14366 & & & & & & & & & \\
\hline 22 & 14368 & 5 & Chinamans CK ESE Pottord & QLD & 14 & & 1.116 & 680 & $17 \cdot 24$ & $145^{\circ} 10^{\prime}$ \\
\hline & 14373 & & & & & & & & & \\
\hline 24 & 14385 & 3 & Emu Ck SE Potford & QLD & 14 & & 873 & 520 & $17 \cdot 26^{\prime}$ & $145 \cdot 02^{\prime}$ \\
\hline & 14387 & & & & & & & & & \\
\hline
\end{tabular}

1.- Temperatura media minima anual $\left({ }^{\circ} \mathrm{C}\right)$

2.- Temperatura media máxima anual $\left({ }^{\circ} \mathrm{C}\right)$

3.- Precipitación media anual $(\mathrm{mm})$

Análisis estadistico

Los valores registrados en cada uno de los ensayos para la variable $d^{2} H$, 
fueron promediados a nivel de procedencias y progenies dentro del ensayo y sometidos a pruebas de análisis de varianza y de comparaciones múltiples de medias (Prueba de Tuckey).

Adicionalmente, se calcularon los promedios generales a nivel de ensayo individual, considerando para esto sólo a las progenies representadas simultáneamente en los cuatro ensayos.

\section{Cuadro $\mathrm{N}^{\circ} 3$}

PROCEDENCIAS Y PROGENIES ESTABLECIDAS EN CADA ENSAYO

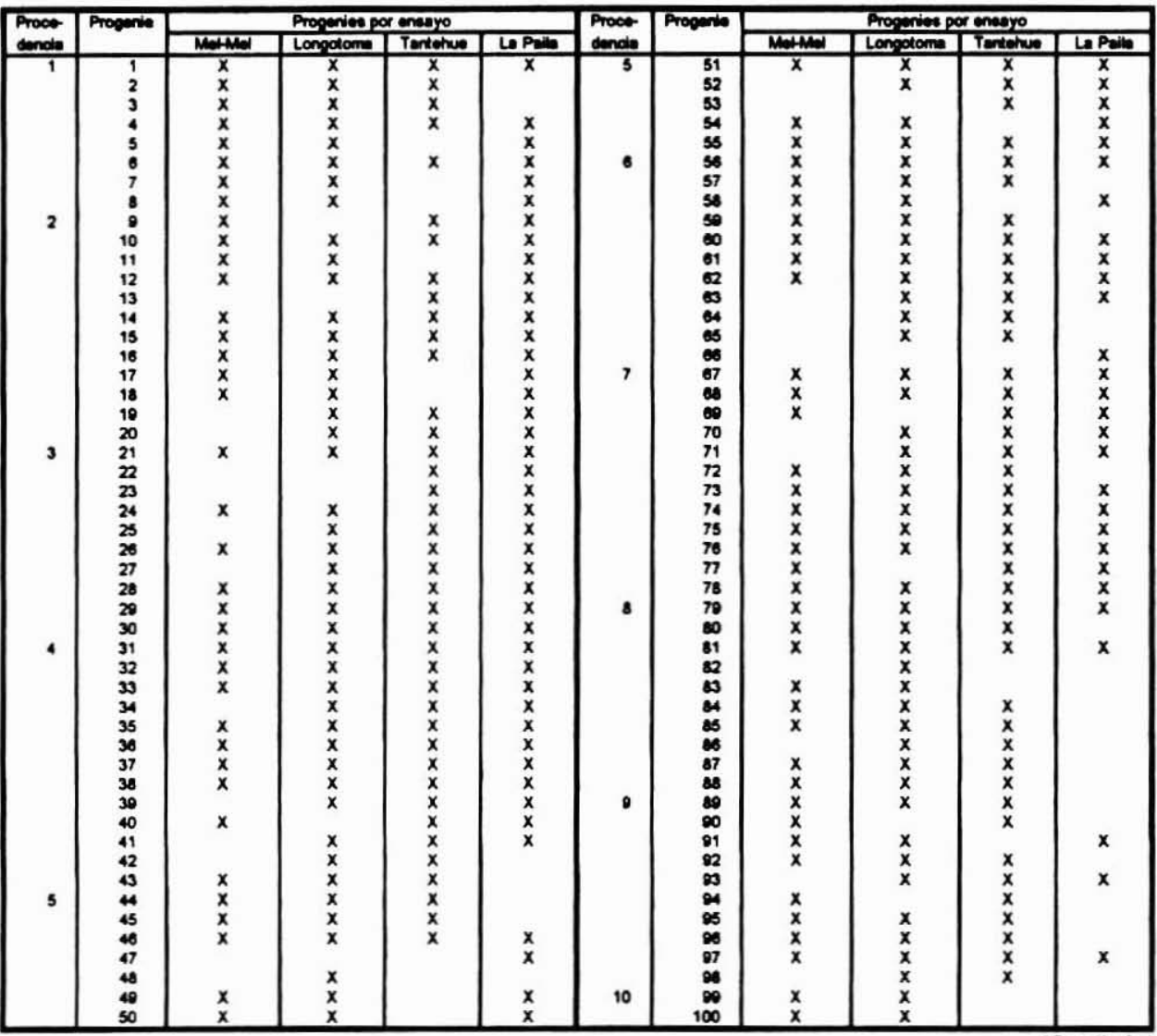

(Continúa) 


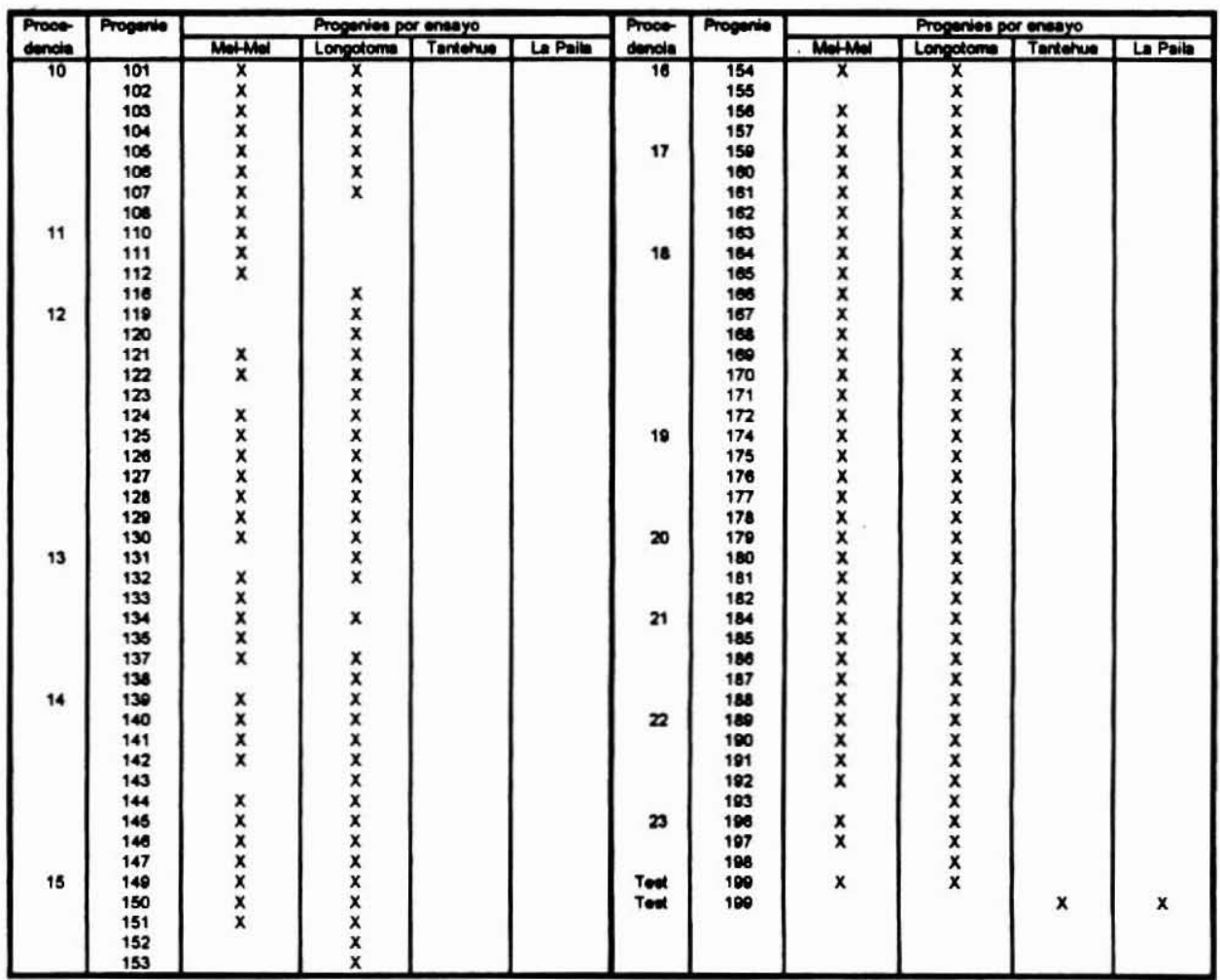

\section{RESULTADOS}

En los Cuadros $N^{\circ}$ s. 4 a 7 se presentan los resultados obtenidos al segundo año de desarrollo de los ensayos, señalándose la supervivencia, altura, diámetro de cuello y $\mathrm{d}^{2} \mathrm{H}$ por procedencia, el rango de variación del $\mathrm{d}^{2} \mathrm{H}$ de las progenies dentro de cada procedencia, el nivel de significación estadística de esta variación y el agrupamiento según Tuckey de las medias por procedencia. 


\section{DISCUSION Y CONCLUSIONES}

\section{Supervivencia}

En los cuatro ensayos analizados, la supervivencia de todas las procedencias consideradas es superior al $90 \%$, existiendo sólo dos excepciones en el ensayo Longotoma, donde la procedencia número 3 (Emu Creek Petford, Queensland) y el testigo nacional (La Ligua), presentan valores de 87,5 y $77,5 \%$, respectivamente.

Las altas tasas de supervivencia registradas en las distintas procedencias son consecuencia de condiciones ambientales favorables para la especie y a la vez confirman la plasticidad de esta.

A pesar de lo anterior, no se debe olvidar que las intensivas técnicas de preparación de suelo para el establecimiento y las labores posteriores, especialmente el control de competencia, han contribuido en forma importante a disminuir la mortalidad de plantas. Por otra parte, aún cuando la sobrevivencia es en general alta, el desarrollo de las distintas procedencia exhibe considerables diferencias entre y dentro de ellas, to que hace que algunas se perfilen como más interesantes que otras, situación que se analizará en el punto siguiente. 


\section{Cuadro $N^{\circ} 4$}

\section{RESULTADOS ENSAYO LONGOTOMA}

\begin{tabular}{|c|c|c|c|c|c|c|c|c|c|}
\hline $\begin{array}{l}\text { Proce- } \\
\text { dencia }\end{array}$ & Lugar de origen & & $\begin{array}{l}H \\
(m)\end{array}$ & $\begin{array}{c}D \\
(\mathrm{~cm})\end{array}$ & $\begin{array}{l}S \\
(\%)\end{array}$ & $\begin{array}{l}d^{2} \mathrm{H} \\
\left(\mathrm{cm}^{3}\right)\end{array}$ & $\begin{array}{l}\text { Test de } \\
\text { Tuckey }\end{array}$ & $\begin{array}{l}\text { Rango } \mathrm{d}^{2} \mathrm{H} \\
\left(\mathrm{cm}^{3}\right)\end{array}$ & (1) \\
\hline 3 & Lake Hindmarsh-SE & VIC & 1,49 & 2,22 & 96,25 & $1.134,30$ & & $480,69-1.605,95$ & \\
\hline 5 & Lake Albacutya-S & VIC & 1,45 & 2,20 & 98,75 & 987,55 & & $393,46-1.596,00$ & $x \alpha$ \\
\hline 4 & Outbet Creek-Rainbow & VIC & 1,41 & 2,09 & 98,08 & 912,46 & & $512,13-1.767,43$ & $x \alpha$ \\
\hline 8 & Lowan Valley-Saline & VIC & 1,23 & 2,08 & 94,50 & 824,58 & & $449,46-1.563,98$ & $x x$ \\
\hline 7 & Lake Albacutya-N & VIC & 1,24 & 2.07 & 98,25 & 785,23 & & $560,09-1.071,49$ & \\
\hline 9 & Avon River-Navarre & VIC & 0,96 & 1,91 & 93,75 & 678,72 & & $195,93-1.409,95$ & $x x$ \\
\hline 6 & Lake Abacutya-E & VIC & 1,21 & 1,94 & 97,00 & 673,73 & & $409,25-1.072,89$ & $x x$ \\
\hline 19 & Emu CK E Potford & OLD & 1,29 & 1,74 & 95,00 & 646,42 & & 475,52 & \\
\hline 21 & Gibbs CK ESE Petford & OLD & 1,25 & 1,82 & 95,50 & 638,84 & & 534,19 - & \\
\hline 2 & Wimmera R-Glenorchy & VIC & 1,08 & 1,98 & 97,73 & 613,91 & & 265,39 - & $x$ \\
\hline 20 & Emu/Gibbs Ck SE Petford & QLD & 1,28 & 1,78 & 99,34 & 580,69 & & $503,30-$ & \\
\hline 1 & Wimmera R-Elmhurst & VIC & 1,08 & 1,95 & 95,00 & 565,43 & & $377,66-$ & $x$ \\
\hline 24 & Emu CK SE Petford & QLD & 1,23 & 1,63 & 87,50 & 561,78 & & $391,80-$ & \\
\hline 12 & Katherine & NT & 1,15 & 1,76 & 96,04 & 518,51 & & 346,89 - & \\
\hline 18 & Hales Siding E Pettord & QLD & 1,23 & 1,70 & 97,50 & 505,30 & & 355,43 - & \\
\hline 17 & Eureka CK NE Petford & QLD & 1,21 & 1,60 & 97,00 & 460,15 & & 338,49 - & \\
\hline 22 & Chinamans CK ESE Petford & OLD & 1.14 & 1,55 & 94,50 & 458,32 & & 313,92 - & \\
\hline 14 & Eccles CK NE Patford & QLD & 1,20 & 1,55 & 96,11 & 407,07 & & 265,64 - & $x$ \\
\hline 15 & Pinnacle CK NE Petford & OLD & 1,15 & 1,50 & 98,50 & 389,85 & & 296,97 - & \\
\hline 13 & Katherine & NT & 1,02 & 1,58 & 94,50 & 357,84 & & 267,30 & \\
\hline 11 & $24 \mathrm{~km}$ Frm Port Lincoln & SA & 0,81 & 1,82 & 95,00 & 325,06 & & 325.06 & \\
\hline 16 & Mishap CK NE Petford & QLD & 1,08 & 1,32 & 95,00 & 297,42 & & $235,40-\quad 395,65$ & \\
\hline 10 & Umberumberka Creek & NSW & 0,82 & 1,55 & 96,39 & 263,18 & & $185,25-$ & \\
\hline Test & La Liqua & Chile & 0,81 & 1,40 & 77,50 & 221,01 & & 221,01 . & \\
\hline
\end{tabular}

(1) Sin diferencia significativa entre progenies dentro de la procedencia.

$X=$ Diferencias significativas $(0,05)$

$X X=$ Diferencias significativas $(0,01)$ 


\section{Cuadro $\mathrm{N}^{\circ} 5$}

RESULTADOS ENSAYO MEL-MEL

\begin{tabular}{|c|c|c|c|c|c|c|c|c|c|c|}
\hline $\begin{array}{l}\text { Proce- } \\
\text { dencia }\end{array}$ & Lugar de origen & & $\begin{array}{c}\mathrm{H} \\
(\mathrm{m})\end{array}$ & $\begin{array}{c}D \\
(\mathrm{~cm})\end{array}$ & $\begin{array}{c}S \\
(\%)\end{array}$ & $\begin{array}{l}\mathrm{d}^{2} \mathrm{H} \\
\left(\mathrm{cm}^{3}\right)\end{array}$ & $\begin{array}{l}\text { Test de } \\
\text { Tuckey }\end{array}$ & \multicolumn{2}{|c|}{$\begin{array}{c}\text { Rango } \mathrm{d}^{2} \mathrm{H} \\
\left(\mathrm{cm}^{3}\right)\end{array}$} & (1) \\
\hline 3 & Lake Hindmarsh-SE & VIC & 1,49 & 2,34 & 96,25 & $1.161,28$ & & 715,57 . & $1.684,68$ & $x \alpha$ \\
\hline 4 & Outlet Croek-Rainbow & VIC & 1,51 & 2,35 & 98,89 & $1.100,99$ & & 783,64 - & $1.662,03$ & $x x$ \\
\hline 5 & Lake Abacutya-S & VIC & 1,40 & 2,16 & 97,50 & 854,44 & | & 586,83 - & $1.179,81$ & $x x$ \\
\hline 7 & Lake Nbacutya-N & VIC & 1,33 & 2,18 & 100,00 & 823,45 & & 572,80 - & $1.157,01$ & \\
\hline 6 & Lake Abacutya-E & & 1,38 & 2,18 & 96,43 & 817,88 & & 505,76 - & 979,92 & $x$ \\
\hline 8 & Lowan Valley-Saline & VIC & 1,34 & 2,12 & 99,06 & 802,23 & & 482,24 - & $1.088,23$ & $x x$ \\
\hline 1 & Wimmera R-Elmhurst & VIC & 1,19 & 2,06 & 98,57 & 658,62 & & $467,49-$ & 935,76 & \\
\hline 9 & Avon River-Navarre & VIC & 1.11 & 2,08 & 98,93 & 628,36 & & 308,07 . & 884,05 & $x \alpha$ \\
\hline 2 & Wimmera R-Glenorchy & VIC & 1.08 & 2,02 & 99,29 & 567,41 & - & 355,26 - & 947,12 & \\
\hline 22 & Chinamans CK ESE Pettord & QLD & 1,23 & 1,68 & 95,63 & 548,62 & & 418,24 - & 774,70 & $x$ \\
\hline 16 & Mishap Ck NE Potford & QLD & 1.11 & 1,63 & 95,83 & 542,21 & & 246,00 - & 797,90 & $x$ \\
\hline 17 & Eureka CK NE Potford & QLD & 1.25 & 1,70 & 99,00 & 481,48 & & 397.57 & 518,90 & \\
\hline 18 & Hales Siding E Potford & QLD & 1.16 & 1,67 & 97,19 & 465,26 & & 293,28 . & 897,31 & \\
\hline 14 & Eccles CK NE Petford & OLD & 1,17 & 1,69 & 98,44 & 442,10 & & 324,97 . & 652,14 & $x \alpha$ \\
\hline 12 & Katherine & NT & 1,07 & 1,70 & 98,06 & 416,83 & & 248,22 & 549,20 & $x$ \\
\hline 21 & Gibbs CK ESE Potford & OLD & 1,14 & 1,68 & 99,00 & 408,02 & & 309,81 . & 559,48 & $x$ \\
\hline 19 & Emu CKE Potford & QLD & 1,20 & 1,56 & 97,50 & 372,91 & & 305,06 . & 441,21 & \\
\hline 24 & Emu CK SE Petford & QLD & 1.24 & 1,55 & 100,00 & 360,73 & & 342,85 & 378,61 & \\
\hline 15 & Pinnacle Ck NE Petford & QLD & 1,11 & 1,56 & 96,67 & 356,78 & & 301,58 . & 410,97 & \\
\hline 11 & $24 \mathrm{~km}$ Fm Port Lincoln & $S A$ & 0,85 & 1,89 & 100,00 & 340,94 & & 245,41 . & 444,81 & \\
\hline 20 & Emu/Gibbs CK SE Petford & OLD & 1,17 & 1,51 & 98,75 & 327,30 & & 277,42 . & 400,96 & \\
\hline 13 & Katherine & NT & 0,94 & 1,54 & 98,75 & 304,59 & & 217,79 & 362,03 & \\
\hline Test & La Ligua & Chile & 0,82 & 1,53 & 92,50 & 275,95 & & 275,95 . & 275,95 & \\
\hline 10 & Umberumberka Creek & NSW & 0,77 & 1,54 & 96,94 & 238.51 & & 176,16 . & 293,91 & \\
\hline
\end{tabular}

(1) Sin diferencia significativa entre progenies dentro de la procedencia.

$X=$ Diferencias significativas $(0,05)$

$X X=$ Diferencias significativas $(0,01)$ 


\section{Cuadro $N^{\circ} 6$}

\section{RESULTADOS ENSAYO TANTEHUE}

\begin{tabular}{|c|c|c|c|c|c|c|c|c|c|c|}
\hline $\begin{array}{l}\text { Proce- } \\
\text { dencia }\end{array}$ & Lugar de origen & & $\begin{array}{c}\mathrm{H} \\
(\mathrm{m}) \\
\end{array}$ & $\begin{array}{c}D \\
(\mathrm{~cm})\end{array}$ & $\begin{array}{c}S \\
(\%)\end{array}$ & $\begin{array}{r}\mathrm{d}^{2} \mathrm{H} \\
\left(\mathrm{cm}^{3}\right) \\
\end{array}$ & $\begin{array}{l}\text { Test de } \\
\text { Tuckey }\end{array}$ & $\begin{array}{c}\text { Rango } \mathrm{d}^{2} \mathrm{r} \\
\left(\mathrm{cm}^{3}\right)\end{array}$ & & (1) \\
\hline 5 & Lale Albacultya-S & VIC & 3,73 & 6,08 & 98,93 & $16.377,13$ & & $10.364,36-$ & 20.366 .87 & $x x$ \\
\hline 9 & Avon River-Navarre & VIC & 3,25 & 5,86 & 98,61 & $14.377,41$ & & $8,440,66$ & $17.397,01$ & $x x$ \\
\hline 6 & Lales Albacutya-E & VIC & 3,48 & 5,73 & 96,94 & $14.245,09$ & & $9.136,62 \cdot$ & $20.471,48$ & $x x$ \\
\hline 3 & Lake Hindmarsh-SE & VIC & 3,53 & 5,72 & 98,00 & $13.844,26$ & & $9.796,88$ - & $17.432,51$ & $x x$ \\
\hline 7 & Lake Albacutya-N & VIC & 3,52 & 5,81 & 98.13 & $13.825,16$ & & $10.911,22$ & $18.622,35$ & $x x$ \\
\hline 8 & Lowan Valley-Saline & VIC & 3,46 & 5,56 & 95,00 & $13.662,51$ & & $5.672,28$ & $16,379,62$ & $x \alpha$ \\
\hline 4 & Outlet Croat-Rainbow & VIC & 3,36 & 5,60 & 99,62 & 12.203 .66 & & $8.638,71$ - & $16.419,41$ & $x \alpha$ \\
\hline 2 & Wimmera R-Glenorchy & VIC & 3,13 & 5.71 & 96,39 & $12.014,77$ & & $9.194,11$. & $14.683,46$ & $x$ \\
\hline 1 & Wimmera R-EImhurst & VIC & 3,16 & 5,41 & 99,50 & $11.679,18$ & & 7890,06 & $15.982,36$ & $x \alpha$ \\
\hline Test & Lolol & Chile & 2,67 & 4,46 & 100,00 & $6.636,96$ & & $6.636,96$. & $6.636,96$ & \\
\hline
\end{tabular}

(1) Sin diferencia significativa entre progenies dentro de la procedencia.

$X=$ Diferencias significativas $(0,05)$

$X X=$ Diferencias significativas $(0,01)$

\section{Cuadro $\mathrm{N}^{\circ} 7$}

\section{RESULTADOS ENSAYO LA PAILA}

\begin{tabular}{|c|c|c|c|c|c|c|c|c|c|c|}
\hline $\begin{array}{l}\text { Proce- } \\
\text { dencia }\end{array}$ & Lugar de origen & & $\begin{array}{c}\mathrm{H} \\
(\mathrm{m}) \\
\end{array}$ & $\begin{array}{c}D \\
(\mathrm{~cm})\end{array}$ & $\begin{array}{c}S \\
(\%)\end{array}$ & $\begin{array}{l}d^{2} \mathrm{H} \\
\left(\mathrm{cm}^{3}\right)\end{array}$ & $\begin{array}{l}\text { Test de } \\
\text { Tuckey }\end{array}$ & \multicolumn{2}{|c|}{$\begin{array}{c}\text { Rango } \mathrm{d}^{2} \mathrm{H} \\
\left(\mathrm{cm}^{3}\right)\end{array}$} & (1) \\
\hline 3 & Lake Hindmarsh-SE & VIC & 1,75 & 2,77 & 93,50 & $2.094,20$ & & 1248.71 . & $2.864,03$ & $x x$ \\
\hline 1 & Wimmera R-Elhurst & VIC & 1.55 & 2.79 & 96,11 & $1.829,04$ & & $1523,85-$ & 2.226 .71 & \\
\hline 7 & Lake Albacutya-N & VIC & 1,64 & 2,59 & 95,68 & $1.483,03$ & 1 & $1.020,79$. & $2.184,50$ & $x$ \\
\hline 6 & Lake Albescutya-E & VIC & 1,54 & 2,45 & 91,15 & 1472,23 & & 868,71 . & $2.381,68$ & $x \alpha$ \\
\hline 5 & Lalos Albacutya-S & VIC & 1,59 & 2,55 & 94,69 & $1.295,84$ & & 961,95 - & $1.845,70$ & $x$ \\
\hline 8 & Lowan Valley-Saline & VIC & 1,55 & 2,45 & 92,50 & $1.241,02$ & & $1.185,04-$ & $1,303,39$ & \\
\hline 2 & Wimmera R-Glenorchy & VIC & 1,38 & 2,47 & 94,44 & 1093,24 & & 599,46 . & $2.393,57$ & $x x$ \\
\hline 4 & Outlet Creek-Rainbow & VIC & 1,46 & 2,33 & 96,74 & $1.054,28$ & & 718,32 - & 1.912 .25 & $x x$ \\
\hline 9 & Avon River-Havarte & VIC & 1,31 & 2,46 & 90,00 & $1.040,02$ & & $922,57 \cdot$ & 1.087 .15 & \\
\hline Test & Lolol & Chile & 1,26 & 1,97 & 95,00 & 622.39 & & 622,39 - & 622,39 & \\
\hline
\end{tabular}

(1) Sin diferencia significativa entre progenies dentro de la procedencia.

$X=$ Diferencias significativas $(0,05)$

$X X=$ Diferencias significativas $(0,01)$ 


\section{Crecimiento}

Se aprecia una clara superioridad en el crecimiento manifestado por las procedencias australianas en relación a las nacionales utilizadas como testigos. Estas últimas exhiben invariablemente los menores valores para la variable $\mathrm{d}^{2} \mathrm{H}$ y sólo en el ensayo de Mel-Mel superan a una procedencia australiana (Umberumberka Creek, NSW), la que a su vez presenta el menor crecimiento dentro de este ensayo.

La variación en crecimiento dentro de las procedencias nacionales no es significativa, lo que sumado al escaso desarrollo que estas manifiestan, permite afirmar que ellas no constituyen un buen material de base para un programa de mejoramiento genético, confirmando a la vez la necesidad de importar semillas desde el área de distribución natural de la especie, para evaluar el desarrollo de una base genética más amplia y, por consiguiente, de mayor variabilidad para ser utilizada en programas de mejoramiento.

El análisis de los valores de $d^{2} \mathrm{H}$ registrados en cada procedencia, permite deducir un patrón general de variación geográfica. En los ensayos de Mel-Mel y Longotoma las procedencias del Estado de Victoria exhiben los mayores crecimientos, posteriormente se encuentran las de Queensland y en último lugar las de New South Wales. Por su parte, las procedencias de Northern y Southern Territory exhiben un patrón de crecimiento irregular y normalmente intermedio entre el de las procedencias de Queensland y New South Wales. Los dos ensayos restantes no contribuyen a este análisis pues sólo consideran procedencias de Victoria.

Las causas de este tipo de comportamiento parecen obedecer, entre otros motivos, a la similitud climática que existe entre las áreas de establecimiento de los ensayos y el lugar de origen de las semillas. En el caso de Victoria, la precipitación media anual, su distribución y la temperatura media son muy similares a las registradas en las zonas de ensayo. Por el contrario, los demás orígenes presentan valores de precipitación considerablemente más altos que los registrados en los lugares de ensayo. Como consecuencia de esto, las poblaciones que han evolucionado en esos lugares poseen requerimientos hídricos mayores a los que pueden satisfacer en las zonas ensayadas y por lo mismo su crecimiento ha sido más deficiente.

A pesar de lo anterior, no se aprecia consistencia en el comportamiento de las distintas procedencias en los cuatro ensayos, esto puede obedecer a que 
ellas no participan con las mismas progenies en cada uno de ellos, 0 a que las características propias de cada sitio favorecen en forma diferencial a las procedencias ensayadas.

Por otra parte, las condiciones de sitio en cada uno de los lugares considerados han motivado importantes diferencias de crecimiento entre ensayos. Efectivamente, los promedios para la variable $\mathrm{d}^{2} \mathrm{H}$ obtenidos con aquellas progenies que se probaron simultáneamente en los cuatro ensayos son; 12.840; 1.420; 893 y 877 cc, correspondiendo a Tantehue, La Paila, Mel-Mel y Longotoma, respectivamente. Aquí se observa que los crecimientos en Mel-Mel y Longotoma son similares y menores a los registrados en La Paila y Tantehue. En el caso particular de este último ensayo, el crecimiento observado es significativamente mayor que en los demás y obedece a condiciones de suelo más favorables, específicamente mayor profundidad, mayor contenido de materia orgánica y mayor humedad producto de la topografia local. Por otra parte, la temporada de establecimiento de los ensayos fue distinta, de modo que las plantas de Longotoma y Mel-Mel debieron soportar un año de sequía, cosa que no ocurrió con los individuos de la Paila y Tantehue, que fueron establecidos en forma posterior, en un año más benigno en materia de lluvias.

Aunque no todas las procedencias fueron consideradas con el mismo número de progenies en los cuatro ensayos, en forma general se puede confirmar que las procedencias de los lagos Hindmarsh y Albacutya exhiben los mejores crecimientos iniciales.

En los ensayos de Mel-Mel y Longotoma, la procedencia Outlet Creek-Rainbow (Victoria) presenta uno de los valores más altos en crecimiento. En los ensayos de Tantehue y La Paila exhibe crecimientos similares 0 significativamente mayores, aún cuando en estas dos últimas situaciones su comportamiento no difiere significativamente de aquellos de las procedencias restantes, e incluso es superado por la mayoría de ellas.

A pesar de que es posible identificar procedencias que presentan buen crecimiento promedio, en la mayoría de los casos la variación que exhiben sus progenies es tan amplia que impide generalizar juicios sobre las procedencias completas. Análogamente, procedencias que en promedio no exhiben crecimientos satisfactorios, presentan progenies que alcanzan valores de gran interés.

La gran variabilidad que registra el crecimiento a nivel de progenies hace que las familias de mayor desarrollo en cada ensayo sean diferentes. Aún así, 
de entre las diez mejores progenies por ensayo algunas son comunes, como es el caso de las familias 22, 24 y 26 de la procedencia Lake Hindmarsh (Victoria), 33 de Outlet Creek-Rainbow (Victoria) y 44 y 69 de las procedencias Sur y Norte, respectivamente, del lago Albacutya (Victoria). En general, estos resultados coinciden con los obtenidos en investigaciones similares y con la experiencia reunida en el Instituto Forestal, coincidiendo en señalar a estas procedencias como las de mayor crecimiento, ya sea inicial o en estados de desarrollo más avanzado.

\section{REFERENCIAS}

Barros, S. 1990. Ensayos de Procedencias de Eucalyptus camaldulensis Denh en la Zona Semiárida de Chile. Ciencia e Investigación Forestal 4(2): 171 - 182.

Bellefontaine, R. et al. 1979. Experience Internationale D'Origines D'Eucalyptus camaldulensis Denhn. Dispositif de Sidi Slimane (Maroc). Ann. de la Recherche Forestiere au Maroc. Vol 19.

Doran, J. 1985. Collection of Eucalyptus camaldulensis Seed from 178 Open Pollinated Families in the Petford Region of North Queensland. CSIRO, Division of Forest Research, Tree Seed Centre. Canberra. $17 \mathrm{p}$.

Eldrige, K. y Cromer, R. 1987. Adaptation and Physiology of Eucalyptus in Relation to Genetic Improvement. En: Actas del Simposio sobre Silvicultura y Mejoramiento Genético de Especies Forestales. Buenos Aires, Argentina. 6 al 10 de abril de 1987

FAO. 1981. Eucalypts for Planting. FAO Forestry Series No. 11. Roma. 677 p.

Infante, P. y Prado, J. 1991. Crecimiento Juvenil de 32 Procedencias y 203 Familias de Eucalyptus globulus ssp globulus en la Zona Costera de la VIII Región de Chile Ciencia e Investigación Forestal 5(2): 251 - 265.

Infante, P. y Prado, J. 1989. Crecimiento de 35 Procedencias de Eucalyptus globulus ssp globulus en Etapa de Vivero. Ciencia e Investigación Forestal 2(7): 83 - 94.

INFOR. 1992. Informe Anual Proyecto: "Investigación en Mejoramiento Genético de Especies del Género Eucalyptus". INFOR, División Silvicultura. S/n pag. 16 anexos. 
INFOR-CORFO. 1986. Especies Forestales Exóticas de Interés Económico para Chile. Santiago, Chile. Gerencia de Desarrollo CORFO, AF 86/32. 167 p.

Knockaert, C. 1984. Production D'Eucalyptus camaldulensis Origine Lake Albacutya Durant la Premiere et la Seconde Rotation. Ann. de la Recherche Forestiere au Maroc. Vol. 24.

Lacaze, J. 1977. Study of the Ecological Adaptation of Eucalyptus: A Study of Provenances of Eucalyptus camaldulensis Denh (FAO Project No. 6). En: Nikles, Burley and Barney. Progress and Problems of Genetic Improvement of Tropical Forest Trees. Vol 22.

Thomson, L. y Merwin, M. 1985. Colecciones de Semilla de Eucalyptus camaldulensis en Victoria Occidental Mayoulunio 1985 FAO, Recursos Genéticos Forestales. Información No. 15. Pp 56 - 60.

Turnbull, J. 1973. Ecología y Variación de Eucalyptus camaldulensis Denh. Instituto de Investigaciones Forestales de Canberra en Información sobre Recursos Genéticos Forestales No. 2. 